\title{
Dynamical properties of non-Markovian stochastic differential equations
}

\author{
A. Hernández-Machado and M. San Miguel \\ Departamento de Física Teórica, Universidad de Barcelona, Diagonal 647, Barcelona-28, Spain
}

(Received 16 November 1982; accepted for publication 17 June 1983)

\begin{abstract}
We study nonstationary non-Markovian processes defined by Langevin-type stochastic differential equations with an Ornstein-Uhlenbeck driving force. We concentrate on the long time limit of the dynamical evolution. We derive an approximate equation for the correlation function of a nonlinear nonstationary non-Markovian process, and we discuss its consequences. Non-Markovicity can introduce a dependence on noise parameters in the dynamics of the correlation function in cases in which it becomes independent of these parameters in the Markovian limit. Several examples are discussed in which the relaxation time increases with respect to the Markovian limit. For a Brownian harmonic oscillator with fluctuating frequency, the non-Markovicity of the process decreases the domain of stability of the system, and it can change an infradamped evolution into an overdamped one.
\end{abstract}

PACS numbers: $05.40 .+\mathrm{j}, 02.50$.Ey

\section{INTRODUCTION}

In this paper we study non-Markovian processes (NM) defined by Langevin-type stochastic differential equations. Several authors ${ }^{1-8,37}$ have discussed the equation satisfied by the probability density of these processes. The knowledge of this quantity is not enough to decide about the Markovicity or non-Markovicity of a process. We focus our attention in quantities like the correlation functions and relaxation times, which give a characterization of the peculiarities of non-Markovian dynamics. These quantities cannot be obtained from the equation for the probability density. We also study non-Markovian effects in the time-dependent moments and in the stability properties of the process. We consider Langevin-type equations without memory kernels and driven by an Ornstein-Uhlenbeck process. The processes solution of this type of equation is nonstationary besides being non-Markovian (NMNS). In the white noise limit of the Ornstein-Uhlenbeck noise, the process becomes simultaneously Markovian and stationary. Nonstationarity is an additional complication which is not present in NM processes described by other equations of the Langevin type. ${ }^{3}$ This paper aims to study the dynamical characterization of those NMNS processes.

The physical motivation of this work is that the type of equation mentioned above is the one used in the description of systems under the influence of external or parametric noise. This description is usually given in terms of phenomenological equations, ${ }^{9}$ in which a parameter is substituted by a random process. This random processes is often modeled by an Ornstein-Uhlenbeck process or in a particular limit by a Gaussian white noise. ${ }^{10,11}$ Such systems have been studied experimentally, ${ }^{10}$ and, in particular, dynamical quantities like relaxation times have been measured. ${ }^{12}$ Our results in this paper describe the dynamical response to an external source of noise coupled to the system. They show the main differences in the dynamical behavior of the system with respect to the Markovian limit in which the OrnsteinUhlenbeck process is replaced by a Gaussian white noise. The dependence of the stationary distribution on the parameters of an Ornstein-Uhlenbeck noise has been determined experimentally. ${ }^{13}$ In the same way it is possible to measure the dependence of a relaxation time on these parameters.

A relevant aspect of this paper is that it gives a practical method that allows an explicit calculation of the dependence of the correlation function and the relaxation time on the parameters of the Ornstein-Uhlenbeck noise. This method is based on ideas similar to the ones used to calculate the probability distribution of these processes ${ }^{5,14,15}$ : It is based on an approximate calculation, by functional methods, of the response of the system to the stochastic driving force. This response function plays a central role in our development. We note that with this method we are able to deal with nonlinear processes, while the practical usefulness of a direct application of more standard approaches based on cumulant expansions seems to be restricted to linear processes.

A central result of this paper is the derivation of an approximate equation for the correlation function of a nonlinear NMNS process. This equation shows that the dynamics of the process depends on the noise parameters in situations in which it is independent of these parameters in the Markovian limit. This is, for example, the case for a process described by a single variable with "additive noise. ${ }^{6 "}$ In this case, the Markovian limit of the equation for the correlation function is noise-independent. This also happens for a Brownian harmonic oscillator with stochastic frequency. Another interesting feature of this equation is the existence of a term which cannot be obtained from the equation for the probability density of the process. This term becomes in some cases the dominant non-Markovian effect. We have found that the relaxation time is increased with respect to the Markovian limit for the most general one-variable model with "additive" or "multiplicative noise 6 " and for the Brownian harmonic oscillator with stochastic frequency. The origin of this common behavior is different in both cases. For the Brownian harmonic oscillator with stochastic frequency, we have also found important non-Markovian effects as the decrease in the range of values of the intensity of frequency fluctuations for which the system is energetically stable and the possibility of a change of dynamic regime from an infradamped to an overdamped mode. This possibility is due to an 
effective reduction of the frequency of oscillation that also appears for a mechanic oscillator with stochastic frequency.

The outline of the paper is as follows. In Sec. 2 we first discuss the peculiarities of the class of nonstationarity nonMarkovian processes that we consider. This is illustrated by means of a simple example. Next we derive approximate equations for the moments and correlation functions of that class of NMNS processes. Sections 3 and 5 are devoted to several examples. We compare our results with those of other approaches. We only consider linear processes (although with multiplicative noise) to concentrate on pure non-Markovian effects independently of nonlinear complications to be studied in future work. Section 3 is devoted to a onevariable model which corresponds to the point reactor kinetic equation. ${ }^{16,17}$ Section 4 considers a general $N$-variable model used in quantum optics. ${ }^{18,19} \mathrm{~A}$ particular case corresponds to the mechanic oscillator with stochastic frequency. ${ }^{20,21}$ In Sec. 5 we study the harmonic Brownian oscillator with stochastic frequency. ${ }^{22-24}$

\section{CORRELATION FUNCTIONS OF NON-MARKOVIAN- NONSTATIONARY PROCESSES}

\section{A. Non-Markovicity and nonstationarity}

Time-convolutionless Fokker-Planck equations for the probability density of NM processes have been considered in Refs. 1-7. It has been pointed out ${ }^{3,5-7}$ that these are not bonafide Fokker-Planck equations because the conditional probability of the process is not a fundamental solution of the Fokker-Planck equation for two arbitrary times. In general, for an NM process, the conditional probability $\alpha\left(q, t ; q^{\prime}, t^{\prime}\right), t>t^{\prime}$, depends not only on $t$ and $t^{\prime}$, but also on the previous history of the process. Only when $t^{\prime}=0$ is $\alpha\left(q, t ; q^{\prime}, 0\right)$ a solution of the Fokker-Planck equation for the probability density $P(q, t)$ with initial condition $P\left(q^{\prime}, 0\right)=\delta\left(q-q^{\prime}\right)$. As a consequence, the Fokker-Planck equation can in general be used to calculate correlation functions like $\langle q(t) q(0)\rangle$ but not $\left\langle q(t) q\left(t^{\prime}\right)\right\rangle$ for arbitrary $t^{\prime}$. Here $t=0$ is taken as the time in which the initial conditions of the NM process are specified. In Ref. 3 an explicit discussion of some of these facts was given considering a Gaussian NM stationary (NMS) process for which exact expressions can be obtained for $\alpha\left(q, t ; q^{\prime}, t^{\prime}\right)$ and $\left\langle q(t) q\left(t^{\prime}\right)\right\rangle$.

We are interested in the calculation of the correlation function and the relaxation time in the steady state of a process solution of a stochastic differential equation of the general form

$$
\dot{q}(t)=v(q(t))+g(q(t)) \xi(t),
$$

where $v(q)$ and $g(q)$ are in general nonlinear functions of $q$. The stochastic force $\xi(t)$ is an Ornstein-Uhlenbeck process: A Gaussian process with zero mean and correlation function

$$
\left\langle\xi(t) \xi\left(t^{\prime}\right)\right\rangle=\gamma\left(t, t^{\prime}\right)=(D / \tau) \exp \left(-\left|t-t^{\prime}\right| / \tau\right) .
$$

A particular process is defined by $(2.1)$ and a given initial condition at the preparation time $t=0$. Any solution of (2.1) is non-Markovian due to the fact that $\xi(t)$ is not a white noise. ${ }^{25}$ For whatever initial condition, the solution of $(2.1)$ is also a nonstationary process. Therefore, Eq. (2.1) defines a class of NMNS processes. These processes are characterized by being a solution of (2.1) and differ from each other in the choice of initial conditions. The nonstationarity gives rise to some difficulties which are not present in the example discussed in Ref. 3 for an NMS process. We note that in the limit $\tau \rightarrow 0,(2.1)$ defines a Markovian process which is also stationary when the distribution of initial conditions is chosen as the stationary distribution. This distribution is the one reached for $t \rightarrow \infty$ and arbitrary initial conditions. Nevertheless, there do not exist two separate limits in which the process becomes NMS and MNS. Therefore, it is important to realize that, in the particular class of processes defined by (2.1), the effects of non-Markovicity and nonstationarity cannot be disentangled. Both have the same origin, which is the finite correlation time $\tau$ of the stochastic force $\xi(t)$. To clarify some features of this class of NMNS processes and the differences with NMS or MNS processes, we first consider a simple explicit example. This example is a Gaussian process defined by $(2.1)$ with

$$
v(q)=-a q, \quad a>0, \quad g(q)=1 .
$$

Following the methods in Refs. 5, 14, and 15 one can immediately obtain the equation satisfied by the probability density of the process $P(q, t)$. This is a time-convolutionless Fokker-Planck equation with time-dependent diffusion coefficient:

$$
\begin{aligned}
& \frac{\partial}{\partial t} P(q, t) \equiv L_{q}(t) P(q, t), \\
& L_{q}(t)=\frac{\partial}{\partial q} a q+D(t) \frac{\partial^{2}}{\partial q^{2}}, \\
& D(t)=\frac{D}{1+\tau a}\left\{1-\exp \left[-\left(\tau^{-1}+a\right) t\right]\right\}
\end{aligned}
$$

The steady-state probability density $P_{s t}(q)$ is obtained as the limit for $t \rightarrow \infty$ of the solution of (2.4) with an arbitrary initial condition: $P_{s t}(q)=\lim _{t \rightarrow \infty} P(q, t)$. This distribution corresponds to the stationary solution of $(2.4)$ when $D(t)$ is replaced by $D(\infty)$.

The intrinsic nonstationarity of (2.1) is explicitly seen in this example: Whatever the initial condition $P(q, t=0)$, the solution of (2.4) depends on time during a transient. In particular, even choosing $P(q, t=0)=P_{s t}(q)$, we obtain a nonstationary process. In the example considered by Fox, ${ }^{3}$ the equation for $P(q, t)$ also features a time-dependent operator $L_{q}(t)$. Nevertheless, the process is stationary when the stationary distribution is chosen as initial condition. Mathematically this happens because the time dependence of $L_{q}(t)$ is only given by a common factor $a(t)$, that is, $L_{q}(t)=a(t) l_{q}$. This does not happen in (2.5). The factorization of the time dependence of $L_{q}(t)$ follows from the assumption of a fluctuation-dissipation relation. In our case (2.1) has to be interpreted as a phenomenological modeling of a system coupled to an external source of noise. This parametric noise is modeled by $\xi(t)$. Therefore, the constant $a$ in (2.3) and the noise parameters $D$ and $\tau$ are assumed to be independent.

In passing, we note that there exists a Markovian nonstationary process (MNS) associated with the NMNS process (2.1), (2.2), and (2.3). It is defined by (2.1) and (2.3) and

$$
\left\langle\xi(t) \xi\left(t^{\prime}\right)\right\rangle=2 D(t) \delta\left(t-t^{\prime}\right)
$$


and $D(t)$ given by (2.6). The bonafide Fokker-Planck equation for this Markovian process is also (2.4). This explicitly shows that Markovicity cannot be decided in terms of the equation satisfied by the probability density. For $\tau \rightarrow 0$ and $D$ fixed, (2.3) and (2.7) define an ordinary Markovian stationary process which is also the Markovian limit of (2.2)-(2.3).

The calculation of the steady-state correlation function of the example (2.2)-(2.3) is straightforward. Integrating $(2.1)-(2.3)$ with arbitrary initial conditions and using (2.2), we have

$$
\begin{aligned}
\left\langle q(t) q\left(t^{\prime}\right)\right\rangle_{\mathrm{st}} & =\lim _{\substack{t, t^{\prime} \rightarrow \infty \\
t-t^{\prime}=s}}\left\langle q(t) q\left(t^{\prime}\right)\right\rangle=\frac{D \tau}{(1+\tau a)(\tau a-1)} \\
& \times\left[\exp \left(\frac{-s}{\tau}\right)-(\tau a)^{-1} \exp (-a s)\right] .
\end{aligned}
$$

We can also consider the quantity $\langle q(s) q(0)\rangle_{\text {st IC }}$. This is defined as the correlation function of the process $(2.3)$ with initial conditions at $t=0$ given by $P_{s t}(q)$. We have

$$
\langle q(s) q(0)\rangle_{\mathrm{st} \mathrm{IC}}=\frac{D}{a(1+\tau a)} \exp (-a s) .
$$

The two quantities $\left\langle q(t) q\left(t^{\prime}\right)\right\rangle_{\mathrm{st}}$ and $\langle q(s) q(0)\rangle_{\mathrm{st}}$ rc are two correlation functions which can be considered for the process defined by $(2.1)-(2.3)$ and $P(q, t=0)=P_{s t}(q)$. They are different because the process is not stationary. Nonstationarity also implies that $\langle q(t+s) q(t)\rangle_{\text {st IC }} \neq\langle q(s) q(0)\rangle_{\text {st IC }}$ for a finite $t^{26}$

The steady-state relaxation time $T_{\mathrm{st}}$ is given by

$$
T_{\mathrm{st}}=\int_{0}^{\infty} d s \frac{\left\langle q(t) q\left(t^{\prime}\right)\right\rangle_{\mathrm{st}}-\langle q\rangle_{\mathrm{st}}^{2}}{\left\langle q^{2}\right\rangle_{\mathrm{st}}-\langle q\rangle_{\mathrm{st}}^{2}}=a^{-1}+\tau
$$

Similarly, a relaxation time can be defined for $\langle q(s) q(0)\rangle_{\text {st IC }}$

$$
T_{0}=\int_{0}^{\infty} d s \frac{\langle q(s) q(0)\rangle_{\mathrm{st} \mathrm{IC}}-\langle q\rangle_{\mathrm{st}}^{2}}{\left\langle q^{2}\right\rangle_{\mathrm{st}}-\langle q\rangle_{\mathrm{st}}^{2}}=a^{-1}
$$

In the Markovian limit of the process $(\tau \rightarrow 0), T_{\mathrm{st}}$ and $T_{0}$ coincide. The dependence of $T_{\mathrm{st}}$ on $\tau$ is a dynamical effect which we study in this paper for the class of NMNS processes defined by (2.1). $T_{0}$ does not depend on $\tau$ because $\langle q(s) q(0)\rangle_{\text {st IC }}$ only depends on $\tau$ through static quantities like $\left\langle q^{2}\right\rangle_{\mathrm{st}}$. This dependence cancels in the calculation of $T_{0}$.

The inequality of the two correlation functions defined above is an important difference with respect to the NMS example studied in Ref. 3. This inequality is a direct consequence of the intrinsic nonstationarity of the process. In fact, it is easy to see that, in general, this inequality holds for any nonstationary process regardless of its Markovian or nonMarkovian character (see below ${ }^{27}$ ). For NMS processes, ${ }^{3}$ the two correlation functions are equal. The existence of this inequality for the NMNS process precludes the use of the operator $L_{q}(t)$ to obtain an equation for $\left\langle q(t) q\left(t^{\prime}\right)\right\rangle_{\mathrm{st}}$. In the following we will derive an approximate equation for this quantity for a general NMNS process specified by (2.1). Let us first summarize the situation for the different cases. We assume that the probability density obeys a Fokker-Planck equation of the form (2.4):

(i) If the process is MNS like the one given by $(2.1)-(2.3)$ and (2.7), then (2.4) is a bonafide Fokker-Planck equation and the conditional probability obeys (2.4) for any $t, t^{\prime}$. Thus

$$
\frac{d}{d t}\left\langle q(t) q\left(t^{\prime}\right)\right\rangle=\left\langle\left(L_{q}^{+} q\right)(t) q\left(t^{\prime}\right)\right\rangle,
$$

where $L_{q}^{+}(t)$ is the adjoint operator of $L_{q}(t)$. This is, of course, true for any Markovian process.

(ii) For an NM process, $\alpha\left(q, s ; q^{\prime}, 0\right)$ is in general a solution of $(2.4)$ :

$$
\frac{\partial}{\partial s} \alpha\left(q, s ; q^{\prime}, 0\right)=L_{q}(s) \alpha\left(q, s ; q^{\prime}, 0\right)
$$

with initial condition $\alpha\left(q, 0 ; q^{\prime}, 0\right)=\delta\left(q-q^{\prime}\right)$. Therefore,

$$
\frac{d}{d s}\langle q(s) q(0)\rangle=\left\langle\left(L_{q}^{+}(s) q(s)\right) q(0)\right\rangle .
$$

This equation is valid for arbitrary initial conditions.

(iii) Consider now that we choose stationary initial conditions and that the process is then stationary (NMS). ${ }^{3}$ For a stationary process we have that $\alpha\left(q, t ; q^{\prime}, t^{\prime}\right)^{\prime}=\alpha\left(q, s ; q^{\prime}, 0\right)$ for arbitrary $t^{\prime}$ and $t=t^{\prime}+s$. Therefore, from (2.13)

$$
\frac{\partial}{\partial t} \alpha\left(q, t ; q^{\prime}, t^{\prime}\right)=L_{q}(s) \alpha\left(q, t ; q^{\prime}, t^{\prime}\right)
$$

with

$$
\alpha\left(q, t^{\prime} ; q^{\prime}, t^{\prime}\right)=\delta\left(q-q^{\prime}\right) .
$$

Thus,

$$
\begin{aligned}
\frac{d}{d t}\left\langle q(t) q\left(t^{\prime}\right)\right\rangle_{\mathrm{st}} & =\left\langle\left(L_{q}^{+}(s) q(t)\right) q\left(t^{\prime}\right)\right\rangle_{\mathrm{st}} \\
& =a(s)\left\langle\left(l_{q}^{+} q\right)(t) q\left(t^{\prime}\right)\right\rangle_{\mathrm{st}},
\end{aligned}
$$

where we have used the relation $L_{q}(t)=a(t) l_{q}$. For stationary initial conditions in (2.14), (2.17) and (2.14) coincide. This is in agreement with the fact that $\langle q(s) q(0)\rangle_{\text {st IC }}$ and $\left\langle q(t) q\left(t^{\prime}\right)\right\rangle_{\mathrm{st}}$ coincide for an NMS process. In the example discussed in Ref. 3 the non-Markovicity of the process is explicitly seen because the solution of $(2.15)-(2.16)$ does not satisfy the Chapman-Kolmogoroff equation.

(iv) For the NMNS process (2.1)-(2.2) Eqs. (2.13) and (2.14) remain valid but the argument made above for an NMS process cannot be repeated here. Therefore, no general equation is known for $\left\langle q(t) q\left(t^{\prime}\right)\right\rangle_{\mathrm{st}}$ up to now in this case.

In conclusion, the steady-state correlation function $\left\langle q(t) q\left(t^{\prime}\right)\right\rangle_{\text {st }}$ cannot be identified with $\langle q(s) q(0)\rangle_{\text {st IC }}$ for a nonstationary process. As a consequence, for an NMNS process we do not have an equation for $\left\langle q(t) q\left(t^{\prime}\right)\right\rangle_{\mathrm{st}}$ expressed only in terms of the Fokker-Planck operator $L_{q}(t)$. From the point of view of the argument above, the difficulty is the nonstationarity of the process. But we remark that for the class of NMNS processes defined by (2.1), nonstationarity cannot be separated from non-Markovicity. Our goal is now to derive an equation for the correlation function $\left\langle q(t) q\left(t^{\prime}\right)\right\rangle_{\mathrm{st}}$ of this class of NMNS processes.

\section{B. Equations for the correlation functions}

In general it is not possible to obtain exact equations for the moments and correlation functions of a process defined by (2.1)-(2.2). Our strategy is then to look for systematic approximation schemes in which the zeroth-order approxi- 
mation is the Markovian limit $\tau=0$. We do that by considering $\tau$ as a small parameter. Our approximation essentially consists in an expansion in powers of $\tau$. This program was already carried out for the equation satisfied by the probability density $P(q, t)$ in Refs. 5, 14, and 15 . From the approximate equation for $P(q, t)$ follows an equation for $\langle q(t)\rangle$. This last equation can be directly obtained as follows: Averaging (2.1) we have

$$
\begin{aligned}
\frac{d}{d t}\langle q(t)\rangle & =\langle v(q(t))\rangle+\langle g(q(t)) \xi(t)\rangle \\
& =\langle v(q(t))\rangle+\int_{0}^{t} d t_{1} \gamma\left(t, t_{1}\right)\left\langle\frac{\delta g(q(t))}{\delta \xi\left(t_{1}\right)}\right\rangle
\end{aligned}
$$

In the last equality we have used the functional characterization of the Gaussian property of $\xi(t){ }^{14,15,28}$ Performing successive partial integrations over $t_{1}$ in (2.18) and using (2.2), we obtain an expansion in powers of $\tau$. This amounts to an approximate calculation of the response function $\delta g(q(t)) /$ $\delta \xi\left(t_{1}\right)$ by expanding around $t_{1}=t$.

We have

$$
\begin{gathered}
\int_{0}^{t} d t_{1} \gamma\left(t, t_{1}\right)\left\langle\frac{\delta g(q(t))}{\delta \xi\left(t_{1}\right)}\right\rangle=\frac{D}{\tau} \exp (-t / \tau)\left\{\left[\tau \exp \left(t_{1} / \tau\right)\right.\right. \\
\left.\cdot\left\langle\frac{\delta g(q(t))}{\delta q(t)} \frac{\delta q(t)}{\delta \xi\left(t_{1}\right)}\right\rangle\right]_{t_{1}=0}^{t_{1}=t}-\left[\tau^{2} \exp \left(\frac{t_{1}}{\tau}\right)\right. \\
\left.\left.\cdot\left\langle\frac{\delta g(q(t))}{\delta q(t)} \frac{d}{d t_{1}}\left(\frac{\delta q(t)}{\delta \xi\left(t_{1}\right)}\right)\right\rangle\right]_{t_{1}=0}^{t_{1}=t}+\cdots\right\} .
\end{gathered}
$$

Recalling that ${ }^{14,15}$

$$
\begin{aligned}
& \left.\frac{\delta q(t)}{\delta \xi\left(t_{1}\right)}\right|_{t_{1} \rightarrow t}=g(q(t)) \\
& \left.\frac{d}{d t_{1}} \frac{\delta q(t)}{\delta \xi\left(t_{1}\right)}\right|_{t_{1}, t}=v(q(t)) \frac{\partial g(q(t))}{\partial q(t)} \\
& -\frac{\partial v(q(t))}{\partial q(t)} g(q(t)) \equiv M(q(t)),
\end{aligned}
$$

we obtain

$$
\begin{aligned}
& \int_{0}^{t} d t_{1} \gamma\left(t, t_{1}\right)\left\langle\frac{\delta g(q(t))}{\delta \xi\left(t_{1}\right)}\right\rangle \\
& \simeq D\left\langle\frac{\partial g(q(t))}{\partial q(t)} g(q(t))\right\rangle-D \tau\left\langle\frac{\partial g(q(t))}{\partial q(t)} M(q(t))\right\rangle \\
& \quad-D \exp \left(-\frac{t}{\tau}\right)\left\langle\left.\frac{\partial g(q(t))}{\partial q(t)} \frac{\delta q(t)}{\delta \xi\left(t_{1}\right)}\right|_{t_{1}=0}\right\rangle
\end{aligned}
$$

where the first terms neglected are proportional to $D \tau^{2}$ and $D \tau e^{-t / \tau}$. The last term in (2.22) contains the quantity $\delta q(t) /$ $\left.\delta \xi\left(t_{1}\right)\right|_{t_{1-0}}$ which is not immediately calculable in general. Nevertheless, this is a transient term that can be safely neglected for $t \gg \tau$. In this approximation and substituting (2.22) in (2.18), we obtain to first order in $\tau$

$$
\begin{aligned}
\frac{d}{d t}\langle q(t)\rangle= & \langle v(q(t))\rangle+D\left\langle\frac{\partial g(q(t))}{\partial q(t)} g(q(t))\right\rangle \\
& -\tau D\left\langle\frac{\partial g(q(t))}{\partial q(t)} M(q(t))\right\rangle .
\end{aligned}
$$

The validity of keeping only terms to first order in $\tau$ in the above $\tau$ expansion depends on the value of $\tau$, the range of values of $q$ of interest and of the particular model. A discussion of this point in a related context is given in Ref. 15. A criterion of validity is obtained comparing the terms of order $\tau$ with the following term in the expansion of order $\tau^{2}$. Past experience with this type of expansion for the calculation of probability densities indicates that it gives good results for reasonably small values of $\tau .^{14}$

The equations for the correlation functions can be obtained along the same lines. Since $q(0)$ is statistically independent of $\xi(t)$ the equation for $\langle q(t) q(0)\rangle$ is derived with no extra complication:

$$
\begin{aligned}
\frac{d}{d s}\langle q(s)\rangle q(0)= & \langle v(q(s)) q(0)\rangle+D\left\langle\frac{\partial g(q(s))}{\partial q(s)} g(q(s)) q(0)\right\rangle \\
& -\tau D\left\langle\frac{\partial g(q(s))}{\partial q(s)} M(q(s)) q(0)\right\rangle .
\end{aligned}
$$

In (2.24) transient terms have also been neglected. Therefore, this equation cannot be used to calculate $T_{0}$ because, by its definition (2.11), $T_{0}$ also depends on the time domain $s \leqslant \tau$.

The identical formal structure of (2.23) and (2.24) is a consequence of the fact that both can be obtained from the equation for $P(q, t)$. The equation for $\left\langle q(t) q\left(t^{\prime}\right)\right\rangle$ requires more care:

$$
\frac{d}{d t}\left\langle q(t) q\left(t^{\prime}\right)\right\rangle=\left\langle v(q(t)) q\left(t^{\prime}\right)\right\rangle+\left\langle g(q(t)) \xi(t) q\left(t^{\prime}\right)\right\rangle .
$$

Proceeding as we did in $(2.18)$

$$
\left\langle g(q(t)) \xi(t) q\left(t^{\prime}\right)\right\rangle=\int_{0}^{t} d t_{1} \gamma\left(t, t_{1}\right)\left\langle\frac{\delta g(q(t))}{\delta \xi\left(t_{1}\right)} q\left(t^{\prime}\right)\right\rangle+\int_{0}^{t^{\prime}} d t_{1} \gamma\left(t, t_{1}\right)\left\langle g(q(t)) \frac{\delta q\left(t^{\prime}\right)}{\delta \xi\left(t_{1}\right)}\right\rangle .
$$

The second term in (2.26) has no counterpart in (2.24) since $\delta q(0) / \delta \xi\left(t_{1}\right)=0$. The two terms in (2.26) can be calculated again by successive partial integrations. For the first term we have

$$
\begin{aligned}
\int_{0}^{t} d t_{1} & \gamma\left(t, t_{1}\right)\left\langle\frac{\delta g(q(t))}{\delta \xi\left(t_{1}\right)} q\left(t^{\prime}\right)\right\rangle \\
= & \int_{0}^{t} d t_{1} \gamma\left(t, t_{1}\right)\left\langle\frac{\partial g(q(t))}{\partial q(t)} \frac{\delta q\left(t^{\prime}\right)}{\delta \xi\left(t_{1}\right)} q\left(t^{\prime}\right)\right\rangle \simeq D\left\langle\left.\frac{\partial g\left(q\left(t^{\prime}\right)\right)}{\partial q(t)} \frac{\delta q(t)}{\delta \xi\left(t_{1}\right)}\right|_{t_{1}=t^{\prime}} q\left(t^{\prime}\right)\right\rangle \\
& -D \tau\left(\left.\frac{\partial g(q(t))}{\partial q(t)}\left(\frac{d}{d t_{1}} \frac{\delta q(t)}{\delta \xi\left(t_{1}\right)}\right)\right|_{t_{1}=t} q\left(t^{\prime}\right)\right\rangle-D \exp \left(-\frac{t}{\tau}\right)\left\langle\left.\frac{\partial g(q(t))}{\partial q(t)} \frac{\delta q(t)}{\delta \xi\left(t_{1}\right)}\right|_{t_{1}=0} q\left(t^{\prime}\right)\right\rangle,
\end{aligned}
$$

where the first terms neglected are proportional to $D \tau^{2}$ and $D \tau e^{-t / \tau}$. The last term in (2.27) is again a transient term that we neglect. Substituting (2.20) and (2.21), we have to first order in $\tau$ 


$$
\int_{0}^{t} d t_{1} \gamma\left(t, t_{1}\right)\left\langle\frac{\delta g(q(t))}{\delta \xi\left(t_{1}\right)} q\left(t^{\prime}\right)\right\rangle=D\left\langle\frac{\partial g(q(t))}{\delta q(t)} g(t) q\left(t^{\prime}\right)\right\rangle-D \tau\left\langle\frac{\partial g(q(t))}{\partial q(t)} M(q(t)) q\left(t^{\prime}\right)\right\rangle
$$

For the second term in (2.26) we have

$$
\begin{aligned}
\int_{0}^{t^{\prime}} d t_{1} \gamma\left(t, t_{1}\right)\left\langle g(q(t)) \frac{\delta q\left(t^{\prime}\right)}{\delta \xi\left(t_{1}\right)}\right\rangle & D \exp \left[-\frac{\left(t-t^{\prime}\right)}{\tau}\right]\left\{\left\langle\left. g(q(t)) \frac{\delta q\left(t^{\prime}\right)}{\delta \xi\left(t_{1}\right)}\right|_{t_{1}=t^{\prime}}\right\rangle-\tau\left\langle\left. g(q(t))\left(\frac{d}{d t_{1}} \frac{\delta q\left(t^{\prime}\right)}{\delta \xi\left(t_{1}\right)}\right)\right|_{t_{1}=t^{\prime}}\right\rangle\right\} \\
& -D \exp \left(-\frac{t}{\tau}\right)\left\langle\left. g(q(t)) \frac{\delta q\left(t^{\prime}\right)}{\delta \xi\left(t_{1}\right)}\right|_{t_{1}=0}\right\rangle,
\end{aligned}
$$

where the first terms neglected are proportional to $D \tau^{2} e^{-\left(t-t^{\prime}\right) / \tau}$ and $D \tau e^{-t / \tau}$. Once again the last term in (2.29) is a transient term that we neglect. We remark that, given the definition (2.8) in which $t, t^{\prime} \rightarrow \infty$, to neglect transient terms in the calculation of $\left\langle q(t) q\left(t^{\prime}\right)\right\rangle_{\mathrm{st}}$ is not an approximation. In the definition (2.10) of $T_{\mathrm{st}}$ we integrate $s=t-t^{\prime}$ from $s=0$ to $s=\infty$, and therefore the first two terms in (2.29) cannot be neglected since we cannot assume that $t-t^{\prime}>\tau$. In an expansion in powers of $\tau$ aimed to calculate $T_{\mathrm{st}}$, the quantity $e^{-\left(t-t^{\prime}\right) / \tau}$ has to be regarded as being of zeroth order in $\tau$. Substituting (2.20) and (2.21) in (2.29), we have to first order

$$
\int_{0}^{t^{\prime}} d t_{1} \gamma\left(t, t_{1}\right)\left\langle g(q(t)) \frac{\delta q\left(t^{\prime}\right)}{\delta \xi\left(t_{1}\right)}\right\rangle=D \exp \left[-\frac{\left(t-t^{\prime}\right)}{\tau}\right]\left\{\left\langle g(q(t)) g\left(q\left(t^{\prime}\right)\right)\right\rangle-\tau\left\langle g(q(t)) M\left(q\left(t^{\prime}\right)\right)\right\rangle\right\} .
$$

From (2.25), (2.26), (2.28), and (2.30) we finally have

$$
\begin{aligned}
\frac{d}{d t}\left\langle q(t) q\left(t^{\prime}\right)\right\rangle_{\mathrm{st}}= & \left\langle v\left(q\left(t^{\prime}\right)\right) q\left(t^{\prime}\right)\right\rangle_{\mathrm{st}}+D\left\langle\frac{\partial g(q(t))}{\partial q\left(t^{\prime}\right)} g(q(t)) q\left(t^{\prime}\right)\right\rangle_{\mathrm{st}}-D \tau\left\langle\frac{\partial g(q(t))}{\partial q(t)} M\left(q\left(t^{\prime}\right)\right) q\left(t^{\prime}\right)\right\rangle_{\mathrm{st}} \\
& +D \exp \left[-\frac{\left(t-t^{\prime}\right)}{\tau}\right]\left\{\left\langle g(q(t)) g\left(q\left(t^{\prime}\right)\right)\right\rangle_{\mathrm{st}}-\tau\left\langle g(q(t)) M\left(q\left(t^{\prime}\right)\right)\right\rangle_{\mathrm{st}}\right\} .
\end{aligned}
$$

This can be rewritten as

$$
\frac{d}{d t}\left\langle q(t) q\left(t^{\prime}\right)\right\rangle_{\mathrm{st}}=\left\langle\left(L_{q}^{+}(\tau) q\right)(t) q\left(t^{\prime}\right)\right\rangle_{\mathrm{st}}+D \exp \left[-\frac{\left(t-t^{\prime}\right)}{\tau}\right]\left\langle g(q(t)) h\left(q\left(t^{\prime}\right)\right)\right\rangle_{\mathrm{st}},
$$

where

$$
h(q)=g(q)-\tau M(q)
$$

and

$$
L_{q}^{+}(\tau)=\left[v(q)+D \frac{\partial g(q)}{\partial q} h(q)\right] \partial_{q}+D g(q) h(q) \partial_{q}^{2}
$$

is the adjoint of the Fokker-Planck operator which appears in the approximate Fokker-Planck equation for the probability density $P(q, t)$ of the process. ${ }^{14,15}$ The comments made about the domain of validity of (2.23) apply also for (2.32). This question is further discussed for a particular example in Sec. 3 and in the Appendix.

The two terms in (2.32) contain in a different way the effects of $\tau$ being $\neq 0$. The existence of the second term indicates that, as discussed in Sec. 2A, the equation for $\left\langle q(t) q\left(t^{\prime}\right)\right\rangle_{\mathrm{st}}$ cannot be obtained from the equation for the probability density. This term dissappears in the limit $\tau \rightarrow 0$. The first term remains in this limit but with $L_{q}^{+}(\tau)$ replaced by the Markovian operator $L_{q}^{+}(\tau=0)$. The second term in (2.32) is the main formal difference with (2.24). As seen in (2.29), it appears because $\xi(t)$ and $q\left(t^{\prime}\right)$ are correlated for $t>t^{\prime}$. This correlation dissappears for $\tau=0$. Such a term vanishes in $(2.22)$ since $\xi(t)$ and $q(0)$ are uncorrelated because the system is prepared at $t=0$.
Equation (2.32) is the starting point for the dynamical characterization of the steady state of the NMNS processes defined by (2.1) and in particular for the calculation of $T_{\mathrm{s}}$. No general statement about the dependence of $T_{\mathrm{st}}$ in $\tau$ can be made due to the nonlinear problem involved in the first term in (2.32). A point to remark is the importance of the contributions coming from the second term in (2.32). This is already seen in the case of additive noise $[g(q)=$ const]: The equation for $\left\langle q(t) q\left(t^{\prime}\right)\right\rangle_{\mathrm{st}}$ only depends on the noise parameters $D$ and $\tau$ through those terms. Therefore, in the Markovian limit, the dynamics of $\left\langle q(t) q\left(t^{\prime}\right)\right\rangle_{\mathrm{st}}$ is independent of the noise parameters but it is not in the NMNS situation. In the Markovian limit $\left\langle q(t) q\left(t^{\prime}\right)\right\rangle_{\mathrm{st}}$ only depends on the noise parameter $D$ through the initial condition $\left\langle q^{2}\right\rangle_{\mathrm{st}}$. Therefore, in this limit, $T_{\mathrm{st}}$ is independent of the noise parameters. The importance of the second term in (2.32) is shown in the simple example (2.1)-(2.3). In that example, $\left\langle g(q(t)) \xi(t) q\left(t^{\prime}\right)\right\rangle$ can be calculated exactly, and one obtains an equation whose 
solution is (2.8). Since $T_{\mathrm{st}}$ is linear in $\tau$ [see (2.10)], the approximate equation (2.32) leads to a correlation function which reproduces exactly the result (2.10). Explicitly, we have

$$
\begin{aligned}
\left\langle g(q(t)) \xi(t) q\left(t^{\prime}\right)\right\rangle & =\left\langle\xi(t) q\left(t^{\prime}\right)\right\rangle \\
= & \frac{D}{1+\tau a}\left\{\exp \left[-\frac{\left(t-t^{\prime}\right)}{\tau}\right]\right. \\
& \left.-\exp \left[-\left(\frac{t}{\tau}+a t^{\prime}\right)\right]\right\} \\
& \simeq D(1-\tau a) \exp \left[-\frac{\left(t-t^{\prime}\right)}{\tau}\right] .
\end{aligned}
$$

The approximate correlation function solution of (2.32) is the expansion of $(2.8)$ to first order in $\tau$. In this simple example $T_{\text {st }}$ is independent of $D$. The important point to remark is that the $\tau$ dependence of $T_{\text {st }}$ has its origin in (2.35) which is the term proportional to $\exp \left[-\left(t-t^{\prime}\right) / \tau\right]$ in $(2.32)$. Therefore, this term originates the $\tau$ dependence of $T_{\mathrm{st}}$ in this example, and it is the dominant non-Markovian effect.

The equations above are easily generalized to the case of several variables $q_{i}, i=1, \ldots, N$. We just quote here the results. The generalizations of (2.1) and (2.2) are

$$
\begin{aligned}
& \dot{q}_{i}(t)=v_{i}(q(t))+g_{i j}(q(t)) \xi_{j}(t), \\
& \left\langle\xi_{i}(t)\right\rangle=0,\left\langle\xi_{i}(t) \xi_{j}\left(t^{\prime}\right)\right\rangle=\frac{D}{\tau_{i}} \delta_{i j} \exp \left[-\frac{\left|t-t^{\prime}\right|}{\tau_{i}}\right] .
\end{aligned}
$$

We obtain

$$
\begin{aligned}
\frac{d}{d t}\left\langle q_{i}(t)\right\rangle= & \left\langle v_{i}(q(t))\right\rangle+D\left\langle\frac{\partial g_{i j}(q(t))}{\partial q_{n}(t)} g_{n j}(q(t))\right\rangle \\
& -D \tau_{j}\left\langle\frac{\partial g_{i j}(q(t))}{\partial q_{n}(t)} M_{n j}(q(t))\right\rangle \\
& -D \tau_{j}\left\langle\frac{\partial g_{i j}(q(t))}{\partial q_{n}(t)} K_{n l j}(q(t)) \xi_{l}(t)\right\rangle
\end{aligned}
$$

where ${ }^{29}$

$$
\begin{aligned}
& M_{n j}=v_{l} \frac{\partial g_{n j}}{\partial q_{l}}-\frac{\partial v_{n}}{\partial q_{l}} g_{l j}, \\
& K_{n l j}=g_{k l} \frac{\partial g_{n j}}{\partial q_{k}}-\frac{\partial g_{n l}}{\partial q_{k}} g_{k j} .
\end{aligned}
$$

The last term in (2.38) has to be further elaborated in each particular case. The equation for $\left\langle q_{i}(t) q_{j}(0)\right\rangle$ is formally identical to (2.38). For $\left\langle q_{i}(t) q_{j}\left(t^{\prime}\right)\right\rangle$ we have ${ }^{30}$

$$
\begin{aligned}
\frac{d}{d t}\left\langle q_{i}(t) q_{j}\left(t^{\prime}\right)\right\rangle= & \left\langle v_{i}(q(t)) q_{j}\left(t^{\prime}\right)\right\rangle \\
& +D\left\langle\frac{\partial g_{i k}(q(t))}{\partial q_{n}(t)} g_{n k}(q(t)) q_{j}\left(t^{\prime}\right)\right\rangle \\
& -D \tau_{k}\left\langle\frac { \partial g _ { i k } ( q ( t ) ) } { \partial q _ { n } ( t ) } \left[ M_{n k}(q(t))\right.\right. \\
& \left.\left.+K_{n l k}(q(t)) \xi_{l}(t)\right] q_{j}\left(t^{\prime}\right)\right\rangle
\end{aligned}
$$

$$
\begin{aligned}
& +D \exp \left[-\frac{\left(t-t^{\prime}\right)}{\tau_{k}}\right]\left\{\left\langle g_{i k}(q(t)) g_{j k}\left(q\left(t^{\prime}\right)\right)\right\rangle\right. \\
& -\tau_{k}\left\langleg _ { i k } ( q ( t ) ) \left[ M_{j k}\left(q\left(t^{\prime}\right)\right)\right.\right. \\
& \left.\left.\left.+K_{j l k}\left(q\left(t^{\prime}\right)\right) \xi_{i}\left(t^{\prime}\right)\right]\right\rangle\right\} .
\end{aligned}
$$

\section{EXAMPLE I: ONE VARIABLE MODEL WITH MULTIPLICATIVE NOISE}

As a first application of the general equations derived above, we consider in this section the model defined by (2.1) and (2.2) with

$$
\begin{aligned}
& v(q)=-a q+c, \\
& g(q)=-b q .
\end{aligned}
$$

$a, b$, and $c$ are constants, and $a$ is chosen to be positive. This is a simple but nontrivial model because of the multiplicative character of the noise $[g(q)$ being nonconstant]. For $c=0$ the model becomes mathematically much simpler, but it then has a degenerate steady state in the sense that $P(q, t) \rightarrow_{t \rightarrow \infty} 0$. Besides its intrinsic interest as an illustrative example, this model is the point reactor kinetic equation used to describe the evolution of the number of neutrons in the presence of parametric noise in a nuclear reactor. ${ }^{16,17}$ For $c=0$ is a one-variable version of models describing the interaction of an atomic system with a laser whose phase fluctuates. ${ }^{18,19}$ The model admits a formal exact solution for $\langle q(t)\rangle,\left\langle q(t) q\left(t^{\prime}\right)\right\rangle{ }^{33}$ Here we concentrate in the calculation in first order in $\tau$ which gives a more direct way of obtaining concrete information. In this approximation we obtain explicit results which illuminate the general discussion of Sec. 2B.

To calculate $T_{\mathrm{st}}$ we need to know $\langle q\rangle_{\mathrm{st}},\left\langle q^{2}\right\rangle_{\mathrm{st}}$, and $\left\langle q(t) q\left(t^{\prime}\right)\right\rangle_{\mathrm{st}}$. Specifying (2.23) to the model (3.1), (3.2) and setting $(d / d t)\langle q(t)\rangle=0$, we have

$$
\langle q\rangle_{\mathrm{st}}=\frac{c\left(1-\tau D b^{2}\right)}{\left(a-D b^{2}\right)}+O\left(\tau^{2}\right) .
$$

An equation for $\left\langle q^{2}(t)\right\rangle$ is obtained following step by step the method used to derive (2.23). We obtain

$$
\begin{aligned}
\frac{d}{d t}\left\langle q^{2}(t)\right\rangle= & -2 a\left\langle q^{2}(t)\right\rangle+2 c\langle q(t)\rangle \\
& +4 D b^{2}\left\langle q^{2}(t)\right\rangle-4 \tau c D b^{2}\langle q(t)\rangle
\end{aligned}
$$

and therefore

$$
\left\langle q^{2}\right\rangle_{\mathrm{st}}=\frac{c^{2}\left(1-3 \tau D b^{2}\right)}{\left(a-2 D b^{2}\right)\left(a-D b^{2}\right)}+O\left\langle\tau^{2}\right) .
$$

We then have to require that $a>2 D b^{2}$ to guarantee the existence of steady-state values of $\left\langle q^{2}\right\rangle$ and $\left\langle q(t) q\left(t^{\prime}\right)\right\rangle .{ }^{34}$ The equation for $\left\langle q(t) q\left(t^{\prime}\right)\right\rangle_{\mathrm{st}}$ follows directly from (2.32) and (3.1)-(3.2) (see the Appendix). Its solution can be written to first order in $\tau$ as

$$
\begin{aligned}
\left\langle q(t) q\left(t^{\prime}\right)\right\rangle_{\mathrm{st}}= & \left(\left\langle q^{2}\right\rangle_{\mathrm{st}}-\langle q\rangle_{\mathrm{st}}^{2}\right) \exp \left[\left(-a+D b^{2}\right)\left(t-t^{\prime}\right)\right] \\
& +\langle q\rangle_{\mathrm{st}}^{2}+\tau D b^{2}\left\{1-\exp \left[-\frac{\left(t-t^{\prime}\right)}{\tau}\right]\right\} \\
& \times \exp \left[\left(-a+D b^{2}\right)\left(t-t^{\prime}\right)\right]\left\langle q^{2}\right\rangle_{\mathrm{st}} \\
& -\tau D b^{2} \exp \left[-\frac{\left(t-t^{\prime}\right)}{\tau}\right] \\
& \times\left[1-\exp \left[\left(-a+D b^{2}\right)\left(t-t^{\prime}\right)\right]\right]\langle q\rangle_{\mathrm{st}}^{2}
\end{aligned}
$$


A criterion for the validity of Eq. (2.32) for $\left\langle q(t) q\left(t^{\prime}\right)\right\rangle_{\mathrm{st}}$ can be given in this example by comparing the terms of order $\tau$ kept in (2.32) with the terms of order $\tau^{2}$, which are neglected. This comparison is made in the Appendix. We obtain for this model that to justify the approximation it must be $\tau a<1$.

From the definition of $T_{\mathrm{st}},(2.10),(3.3),(3.5)$, and (3.6), we obtain to first order in $\tau$

$$
T_{\mathrm{st}}=\frac{1}{a-D b^{2}}+\tau+O\left(\tau^{2}\right)
$$

The quantity $1 /\left(a-D b^{2}\right)$ is the Markovian relaxation time. Therefore, we obtain the same result as that in (2.10): For a small $\tau \neq 0$ a contribution $\tau$ is added to the Markovian value of $T_{\mathrm{st}}$. It is easy to see that the terms proportional to $\tau \exp \left[-\left(t-t^{\prime}\right) / \tau\right]$ in (3.6) contribute in order $\tau^{2}$ to $T_{\mathrm{st}}$. The $\tau$ contribution comes from the term in (3.6) proportional to $\tau$. This term has its origin in the terms proportional to $\exp \left[-\left(t-t^{\prime}\right) / \tau\right]$ in $(2.32)$. Thus we conclude that for linear processes with additive or multiplicative noise $T_{\mathrm{st}}$ depends additively on $\tau$ (for small $\tau$ ) and that this dependence is obtained from the second term in (2.32). For nonlinear processes the first term in (2.32) will also contribute to the $\tau$ dependence of $T_{\mathrm{st}}$.

In the simpler case with $c=0$ it is possible to write explicit exact equations for $\langle q(t)\rangle$ and $\left\langle q(t) q\left(t^{\prime}\right)\right\rangle$. From $(2.18)$ we have

$$
\frac{d}{d t}\langle q(t)\rangle=-a\langle q(t)\rangle-b \int_{0}^{t} d t_{1}\left\langle\frac{\delta q(t)}{\delta \xi\left(t_{1}\right)}\right\rangle \gamma\left(t, t_{1}\right) .
$$

The response function is calculated from the integral form of (2.1), (3.1), and (3.2) with $c=0$ :

$$
\frac{\delta q(t)}{\delta \xi\left(t_{1}\right)}=-b q(t) \text {. }
$$

Substituting in (3.8) (see also Ref. 35)

$$
\frac{d}{d t}\langle q(t)\rangle=-a\langle q(t)\rangle+D b^{2}\left[1-\exp \left(-\frac{t}{\tau}\right)\right]\langle q(t)\rangle .
$$

Similarly, substituting (3.9) in (2.25) and (2.26), we obtain an exact equation for $\left\langle q(t) q\left(t^{\prime}\right)\right\rangle$ :

$$
\begin{aligned}
& \frac{d}{d t}\left\langle q(t) q\left(t^{\prime}\right)\right\rangle \\
& =-a\left\langle q(t) q\left(t^{\prime}\right)\right\rangle+D b^{2}\{1-2 \exp (-t / \tau) \\
& \left.\quad+\exp \left[-\left(t-t^{\prime}\right) / \tau\right]\right\}\left\langle q(t) q\left(t^{\prime}\right)\right\rangle .
\end{aligned}
$$

This equation is in agreement with the one obtained in Ref. 36.

\section{EXAMPLE II. N-VARIABLE MODEL WITH MULTIPLICATIVE NOISE}

We consider here a particular example of $(2.36)$ and (2.37) given by

$$
\dot{q}_{i}(t)=-\sum_{j} A_{i j} q_{j}(t)-\sum_{j} B_{i j} q_{j}(t) \xi(t), \quad i j=1, \ldots, N .
$$

$A$ and $B$ are in general noncommuting matrices and $\xi(t)$ is the Ornstein-Uhlenbeck process defined in (2.2). The model is a generalization to $N$ variables of $(3.1)-(3.2)$ with $c=0$. The complications that appear in this generalization are due to the noncommutativity of $A$ and $B$. As already mentioned, (4.1) is a general model used in quantum optics. ${ }^{18,19}$ For a specific choice of $A$ and $B,(4.1)$ also describes the evolution in phase space of a mechanic oscillator whose frequency fluctuates around a mean value. ${ }^{20.21}$ As we did in Sec. 3, we concentrate here on the calculation in first order in $\tau$.

An approximate equation for $\left\langle q_{i}(t)\right\rangle$ follows from (2.38):

$$
\begin{aligned}
\frac{d}{d t}\left\langle q_{i}(t)\right\rangle= & \sum_{j, k}\left(-A_{i j}+D B_{i k} B_{k j}\right. \\
& \left.-\tau D B_{i k}[A, B]_{k j}\right)\left\langle q_{j}(t)\right\rangle+O\left(\tau^{2}\right),
\end{aligned}
$$

where $[A, B]=A B-B A$. It is interesting to note that the non-Markovian contribution proportional to $\tau$ only appears when $A$ and $B$ do not commute. If $A$ and $B$ commute, no contribution to any order in $\tau$ appears in the equation for $\left\langle q_{i}(t)\right\rangle$. For $[A, B]=0$ the exact equation is a trivial generalization of (3.10):

$$
\begin{aligned}
\frac{d}{d t}\left\langle q_{i}(t)\right\rangle= & \sum_{j, k}\left(-A_{i j}+D B_{i k} B_{k j}\right. \\
& \left.\times\left[1-\exp \left(-\frac{t}{\tau}\right)\right]\right)\left\langle q_{j}(t)\right\rangle .
\end{aligned}
$$

A different approximation scheme than ours has been proposed in Ref. 18 to obtain an equation for $\left\langle q_{i}(t)\right\rangle$. The first-order equation in such a scheme has been interpreted in terms of an approximation of Bourret's equation. ${ }^{19}$ In comparison with our scheme we wish to point out that the firstorder approximation in Ref. 18 does not correspond to a first-order approximation in $\tau$. Indeed, when $[A, B]=0$, the first-order equation of Ref. 18 still contains a correction to the Markovian equation $(\tau=0)$. Such a term does not appear either in the exact equation (4.3) or in our approximation (4.2). On the other hand, Van Kampen's approximation ${ }^{8}$ discussed for this model in Ref. 19 reproduces the exact equation (4.3) when $[A, B]=0$. For $[A, B] \neq 0$ an expansion to first order in $\tau$ of van Kampen's equation leads to our result (4.2).

We now consider the equations for the correlation function. The equation for $\left\langle q_{i}(t) q_{j}(0)\right\rangle$ is formally identical to (4.2). An approximate equation for the correlation function $\left\langle q_{i}(t) q_{j}\left(t^{\prime}\right)\right)$ follows from $(2.41)$ :

$$
\begin{aligned}
\frac{d}{d t}\left\langle q_{i}(t) q_{j}\left(t^{\prime}\right)\right\rangle= & \sum_{k, l}\left(-A_{i k}+D B_{i l} B_{l k}\right. \\
& \left.-\tau D B_{i l}[A, B]_{l k}\right)\left\langle q_{k}(t) q_{j}\left(t^{\prime}\right)\right\rangle \\
& +\sum_{k l} D \exp \left[-\frac{\left(t-t^{\prime}\right)}{\tau}\right]\left(B_{i k} B_{j l}\right. \\
& \left.+\tau B_{i k}[B, A]_{j l}\right)\left\langle q_{k}(t) q_{l}\left(t^{\prime}\right)\right\rangle .
\end{aligned}
$$

This equation is only valid for long times since transient terms have been neglected. In particular, if a steady state exists, (4.4) becomes an equation for $\left\langle q_{i}(t) q_{j}\left(t^{\prime}\right)\right\rangle_{\mathrm{st}}$.

A particular case of (4.1) is given by a mechanic oscillator with position $q$, momentum $p$, unit mass and stochastic frequency $\left[\Omega_{0}^{2}+\xi(t)\right]^{1 / 2}$ with mean value $\Omega_{0}$ : 


$$
\begin{aligned}
& \dot{q}(t)=p(t), \\
& \dot{p}(t)=-\left[\Omega_{0}^{2}+\xi(t)\right] q(t) .
\end{aligned}
$$

The matrices $A, B$, and $[A, B]$ are in this case

$$
\begin{aligned}
& A=\left(\begin{array}{rr}
0 & -1 \\
\Omega_{0}^{2} & 1
\end{array}\right), B=\left(\begin{array}{ll}
0 & 0 \\
1 & 0
\end{array}\right), \\
& {[A, B]=\left(\begin{array}{rr}
-1 & 0 \\
0 & 1
\end{array}\right) .}
\end{aligned}
$$

Equation (4.2) becomes

$$
\begin{aligned}
& \frac{d}{d t}\langle q(t)\rangle=\langle p(t)\rangle, \\
& \frac{d}{d t}\langle p(t)\rangle=\left(-\Omega_{0}^{2}+D \tau\right)\langle q(t)\rangle .
\end{aligned}
$$

This means that for small $\tau$ and $t \gg \tau$ the mean values oscillate with a frequency $\omega=\left(\Omega_{0}^{2}-D \tau\right)^{1 / 2}$. This effective reduction of the frequency of oscillation is a non-Markovian effect which disappears in the limit $\tau \rightarrow 0$. For white noise frequency fluctuations, $\langle q(t)\rangle$ and $\langle p(t)\rangle$ oscillate with the mean frequency $\Omega_{0}$ independently of the noise intensity $D$.

The equations for the correlation functions are easily written from (4.4) and (4.7)-(4.8).

\section{BROWNIAN OSCILLATOR WITH FLUCTUATION FREQUENCY}

The stochastic differential equations that describe the evolution of the position $q$ and momentum $p$ of a Brownian harmonic oscillator of unit mass and fluctuating frequency $\operatorname{are}^{22-24,37}$

$$
\begin{aligned}
& \dot{q}(t)=p(t), \\
& \dot{p}(t)=-2 \lambda p(t)-\left[\Omega_{0}^{2}+\xi(t)\right] q(t)+\eta(t) .
\end{aligned}
$$

Here $\Omega_{0}$ is the mean value of the frequency and $\xi(t)$ the fluctuating part of $\Omega^{2}$. This fluctuating part is modeled by the Ornstein-Uhlenbeck process (2.2). The damping coefficient is $2 \lambda$ and $\eta(t)$ is the thermal noise assumed to be Gaussian white noise with zero mean value and satisfying the usual fluctuation-dissipation relation:

$$
\left\langle\eta(t) \eta\left(t^{\prime}\right)\right\rangle=4 \lambda K_{B} T \delta\left(t-t^{\prime}\right) .
$$

The process (5.1) and (5.2) is NMNS due to the fact that $\xi(t)$ is not a white noise. In the following we study non-Markovian dynamical effects (to leading order in the correlation time $\tau$ ) in the equations for average values, in the stability properties of the system, correlation functions and relaxation time.

Equations (5.1)-(5.2) are a particular case of (2.36) in which $\xi_{1}=\xi$ and $\xi_{2}=\eta\left(\tau_{2}=0\right)$.

The approximate equation for the first moments follow from (2.38):

$$
\begin{aligned}
& \frac{d}{d t}\langle q(t)\rangle=\langle p(t)\rangle, \\
& \frac{d}{d t}\langle p(t)\rangle=\left(-\Omega_{0}^{2}+D \tau\right)\langle q(t)\rangle-2 \lambda\langle p(t)\rangle .
\end{aligned}
$$

The effective reduction of the frequency is the effect already found in (4.10) but here has an important consequence: The solution $\langle q(t)\rangle$ of $(5.4)-(5.5)$ decays to zero in an infradamped oscillatory mode when $\lambda^{2}<\Omega_{0}^{2}-D \tau$. It decays to zero in an overdamped mode when $\lambda^{2}>\Omega^{2}-D \tau$. Therefore, changing the degree of non-Markovicity measured by $\tau$ it is possible to change the regime of the oscillator from the overdamped to the infradamped mode and vice versa when $\lambda^{2} \simeq \Omega_{0}^{2}$.

The equations for the second-order moments are easily obtained by means of the $\tau$ expansion used in Sec. 2B. We find

$$
\begin{aligned}
& \langle q(t) \eta(t)\rangle=0, \\
& \langle p(t) \eta(t)\rangle=2 \lambda K_{B} T \\
& \left\langle\xi(t) q^{2}(t)\right\rangle=-2 D \tau\left\langle q^{2}(t)\right\rangle+O\left(\tau^{2}\right), \\
& \langle\xi(t) q(t) p(t)\rangle=-D(1-2 \lambda \tau)\left\langle q^{2}(t)\right\rangle+O\left(\tau^{2}\right) .
\end{aligned}
$$

Therefore, the approximate equations to first order in $\tau$ are

$$
\begin{aligned}
\frac{d}{d t}\left\langle q^{2}(t)\right\rangle= & 2\langle p(t) q(t)\rangle, \\
\frac{d}{d t}\langle p(t) q(t)\rangle= & -\left(\Omega_{0}^{2}-2 D \tau\right)\left\langle q^{2}(t)\right\rangle \\
& +\left\langle p^{2}(t)\right\rangle-2 \lambda\langle p(t) q(t)\rangle, \\
\frac{d}{d t}\left\langle p^{2}(t)\right\rangle= & 2 D(1-2 \lambda \tau)\left\langle q^{2}(t)\right\rangle-4 \lambda\left\langle p^{2}(t)\right\rangle \\
& -2 \Omega_{0}^{2}\langle p(t) q(t)\rangle+4 \lambda K_{B} T .
\end{aligned}
$$

If a stationary-state value exists for the second-order moments, this is given by the stationary solution of $(5.10)-$ (5.12)

$$
\begin{aligned}
& \langle p q\rangle_{\mathrm{st}}=0, \\
& \left\langle p^{2}\right\rangle_{\mathrm{st}}=\left\langle q^{2}\right\rangle_{\mathrm{st}}\left(\Omega_{0}^{2}-2 D \tau\right), \\
& \left\langle q^{2}\right\rangle_{\mathrm{st}}=\frac{2 \lambda K_{B} T}{2 \lambda \Omega_{0}^{2}-D(2 \lambda \tau+1)} .
\end{aligned}
$$

Equation (5.15) indicates that a finite stationary value of $\left\langle q^{2}\right\rangle$ is not reached for $t \rightarrow \infty$ when $2 \lambda \Omega_{0}^{2}-D(2 \lambda \tau+1)<0$. We then take the stability limit as $D=D_{c}$, where

$$
D_{c}=\frac{2 \lambda \Omega_{0}^{2}}{2 \lambda \tau+1} \simeq 2 \lambda \Omega_{0}^{2}(1-2 \lambda \tau) .
$$

For $D>D_{c}$ the oscillator is energetically unstable. It is easy to check that the condition $D<D_{c}$ guarantees that the linear system $(5.10)-(5.12)$ has no real positive eigenvalue. The nonMarkovicity of the process decreases the stability of the system in the sense that $D_{c}$ is smaller than the critical value of $D$ for $\tau \rightarrow 0$.

We now consider the correlation functions in the steady state. From (2.41) we have

$$
\begin{aligned}
& \frac{d}{d t}\left\langle q(t) q\left(t^{\prime}\right)\right\rangle_{\mathrm{st}}=\left\langle p(t) q\left(t^{\prime}\right)\right\rangle_{\mathrm{st}}, \\
& \begin{aligned}
& \frac{d}{d t}\left\langle p(t) q\left(t^{\prime}\right)\right\rangle_{\mathrm{st}} \\
& \quad\left\{-\Omega_{0}^{2}+D \tau+D \tau \exp \left[-\frac{\left(t-t^{\prime}\right)}{\tau}\right]\right\} \\
& \quad \times\left\langle q(t) q\left(t^{\prime}\right)\right\rangle_{\mathrm{st}}-2 \lambda\left\langle p(t) q\left(t^{\prime}\right)\right\rangle_{\mathrm{st}}, \\
& \frac{d}{d t}\left\langle q(t) p\left(t^{\prime}\right)\right\rangle_{\mathrm{st}}=\left\langle p(t) p\left(t^{\prime}\right)\right\rangle_{\mathrm{st}},
\end{aligned}
\end{aligned}
$$




$$
\begin{aligned}
& \frac{d}{d t}\left\langle p(t) p\left(t^{\prime}\right)\right\rangle_{\mathrm{st}} \\
&= D \exp \left[-\frac{\left(t-t^{\prime}\right)}{\tau}\right](1-2 \lambda \tau)\left\langle q(t) q\left(t^{\prime}\right)\right\rangle_{\mathrm{st}} \\
&+\left\{-\Omega_{\mathrm{o}}^{2}+D \tau-D \tau \exp \left[-\frac{\left(t-t^{\prime}\right)}{\tau}\right]\right\} \\
& \times\left\langle q(t) p\left(t^{\prime}\right)\right\rangle_{\mathrm{st}}-2 \lambda\left\langle p(t) p\left(t^{\prime}\right)\right\rangle_{\mathrm{st}} .
\end{aligned}
$$

The position-position correlation function solution of $(5.17)-(5.20)$ is given in the overdamped mode by

$$
\begin{aligned}
\left\langle q(t) q\left(t^{\prime}\right)\right\rangle_{\mathrm{st}}= & \left\langle q^{2}\right\rangle_{\mathrm{st}} \exp \left[-\lambda\left(t-t^{\prime}\right)\right]\left[\cosh \omega\left(t-t^{\prime}\right)\right. \\
& \left.+(\lambda / 2 \omega) \sinh \omega\left(t-t^{\prime}\right)\right],
\end{aligned}
$$

where $\omega^{2}=\lambda^{2}-\left(\Omega_{0}^{2}-D \tau\right)$. In the infradamped mode we have an oscillatory decay of the correlation function

$$
\begin{aligned}
\left\langle q(t) q\left(t^{\prime}\right)\right\rangle_{\mathrm{st}}= & \left\langle q^{2}\right\rangle_{\mathrm{st}} \exp \left[-\lambda\left(t-t^{\prime}\right)\right]\left[\cos \bar{\omega}\left(t-t^{\prime}\right)\right. \\
& \left.+(\lambda / 2 \bar{\omega}) \sin \bar{\omega}\left(t-t^{\prime}\right)\right],
\end{aligned}
$$

where $\bar{\omega}=\left(\Omega_{0}^{2}-D \tau\right)-\lambda^{2}$. To obtain (5.21) and (5.22), we have neglected the term proportional to $\tau \exp \left[-\left(t-t^{\prime}\right) / \tau\right]$ in (5.18), which gives a contribution to the correlation function of higher order in $\tau$.

Equations (5.21) and (5.22) exhibit an important nonMarkovian dynamical effect: The correlation function depends on the frequency fluctuations not only through the static part $\left\langle q^{2}\right\rangle_{\mathrm{st}}$ but also through the effective frequency $(\omega$ or $\bar{\omega})$ which characterizes the dynamics. In the Markovian limit $\tau \rightarrow 0$, the dynamical evolution of the correlation function is independent of the frequency fluctuations. In this limit the evolution equations for the correlation functions do not depend on the frequency fluctuation. These only come into the expression of $\left\langle q(t) q\left(t^{\prime}\right)\right\rangle_{\mathrm{st}}$ through the initial condition $\left\langle q^{2}\right\rangle_{\mathrm{st}}$. The same effect was already discussed in general after (2.32) for a single variable process with additive noise. Here we have a two-variable process with multiplicative noise such that the Markovian dynamics is noise-independent. Analogously to what happens in (2.10), this non-Markovian dynamical effect manifests itself in a dependence of the relaxation time on the frequency fluctuations. This dependence does not exist in the Markovian limit: The relaxation time of $\left\langle q(t) q\left(t^{\prime}\right)\right\rangle_{\mathrm{st}}$ as defined in (2.10) is, in the overdamped mode,

$$
\begin{aligned}
T_{\mathrm{st}} & =\frac{2 \lambda}{\Omega_{0}^{2}}+\left(\frac{2 \lambda}{\Omega_{0}^{2}}\right)^{2} K_{B} T \tau+O\left(\tau^{2}\right) \\
& =T_{\mathrm{st}}^{w}+\left(T_{s t}^{w}\right)^{2} K_{B} T \tau+O\left(\tau^{2}\right) .
\end{aligned}
$$

Here $T_{\mathrm{st}}^{w}$ is the Markovian relaxation time obtained in the limit $\tau \rightarrow 0$. This result is qualitatively the same that we found in (2.10) and (3.7), that is, an increase of $T_{\mathrm{st}}$ to leading order in $\tau$ with respect to the Markovian limit. Nevertheless, this $\tau$ dependence does not originate here in the terms proportional to $e^{-\left(t-i^{\prime}\right) / \tau}$, which have been neglected in the equations for the correlation functions.

Finally, we wish to compare our results with other studies of the problem of a Brownian harmonic oscillator with fluctuating frequency. In Refs. 23 and 24 the case $\tau=0$ has been studied. Our results reduce to those of Refs. 23 and 24 in this limit. For $\tau \neq 0$ we have found that the dynamics depends on the frequency fluctuations and that the system is energetically less stable. In Ref. $22, \xi(t)$ is taken to be a twostate Markov process instead of an Ornstein-Uhlenbeck noise and in Ref. 21 thermal fluctuations modeled by $\eta(t)$ are neglected. Due to the simpler assumption for $\xi(t)$, an exact stability condition was obtained in Ref. 22 . To first order in the correlation time of the noise, this condition coincides with our result (5.16). The stability condition of Ref. 21 is just the condition (5.16) with $\tau=0$. The effect of a finite $\tau$ does not appear there due to the crudeness of the approximation. There is no explicit expression for the relaxation time in Ref. 22 that could be compared with (5.23).

\section{ACKNOWLEDGMENTS}

We are indebted to J. M. Sancho and L. Pesquera for helpful comments.

\section{APPENDIX}

Equation (2.31) becomes, for the model (3.1)-(3.2),

$$
\begin{aligned}
\frac{d}{d t}\left\langle q(t) q\left(t^{\prime}\right)\right\rangle_{\mathrm{st}}= & \left(-a+D b^{2}\right)\left\langle q(t) q\left(t^{\prime}\right)\right\rangle_{\mathrm{st}} \\
& +c\left(1-\tau D b^{2}\right)\langle q\rangle_{\mathrm{st}} \\
& +D \exp \left[-\frac{\left(t-t^{\prime}\right)}{\tau}\right] \\
& \times\left(b^{2}\left\langle q^{2}\right\rangle_{\mathrm{st}}-\tau b^{2} c\langle q\rangle_{\mathrm{st}}\right) .
\end{aligned}
$$

Integration of (A1) with (3.3) leads to (3.6) in first order in $\tau$.

We now consider the first terms neglected in (2.31) for this case. The first nontransient terms neglected in $(2.27)$ and (2.29) are, respectively,

$$
D \tau^{2}\left\langle\left.\frac{\partial g(q(t))}{\partial q(t)}\left(\frac{d^{2}}{d t_{1}^{2}} \frac{\delta q(t)}{\delta \xi\left(t_{1}\right)}\right)\right|_{t_{1}=t} q\left(t^{\prime}\right)\right\rangle_{\mathrm{st}}
$$

and

$$
D \tau^{2} \exp \left[-\frac{\left(t-t^{\prime}\right)}{\tau}\right]\left\langle\left. g(q(t))\left(\frac{d^{2}}{d t_{1}^{2}} \frac{\delta q\left(t^{\prime}\right)}{\delta \xi\left(t_{1}\right)}\right)\right|_{t_{1}=t^{\prime}}\right\rangle_{\mathrm{st}} .
$$

For the model (3.1)-(3.2) we have

$$
\begin{aligned}
\frac{\delta q(t)}{\delta \xi\left(t_{1}\right)}= & -b \exp \left\{-\int_{0}^{t} d t^{\prime}\left[a+b \xi\left(t^{\prime}\right)\right] q(0)\right\} \\
& -c b \int_{0}^{t_{1}} d t^{\prime} \exp \left\{-\int_{t^{\prime}}^{t} d t^{\prime \prime}\left[a+b \xi\left(t^{\prime \prime}\right)\right]\right\} \\
= & -b q(t)+c b \int_{t_{1}}^{t} d t^{\prime} \\
& \times \exp \left\{-\int_{t^{\prime}}^{t} d t^{\prime \prime}\left[a+b \xi\left(t^{\prime \prime}\right)\right]\right\}
\end{aligned}
$$

and therefore

$$
\left.\frac{d^{2}}{d t_{1}^{2}} \frac{\delta q(t)}{\delta \xi\left(t_{1}\right)}\right|_{t_{1}=t}=-c b[a+b \xi(t)] .
$$

Substituting in (A2) 


$$
\begin{aligned}
(\mathrm{A} 2)= & D \tau^{2} c b^{2}\left[a\langle q\rangle_{\mathrm{st}}+b\left\langle\xi(t) q\left(t^{\prime}\right)\right\rangle_{\mathrm{st}}\right] \\
= & D \tau^{2} c b^{2}\left[a\langle q\rangle_{\mathrm{st}}+b \int_{0}^{t^{\prime}} d t_{1} \gamma\left(t, t_{1}\right)\right. \\
& \left.\times\left\langle\frac{\delta q\left(t^{\prime}\right)}{\delta \xi\left(t_{1}\right)}\right\rangle_{\mathrm{st}}\right] \\
= & D \tau^{2} c b^{2}\left\{a\langle q\rangle_{\mathrm{st}}\right. \\
& \left.-D \exp \left[-\frac{\left(t-t^{\prime}\right)}{\tau}\right] b^{2}\langle q\rangle_{\mathrm{st}}+O(\tau)\right\} .
\end{aligned}
$$

Substituting (A5) in (A3),

$$
\begin{aligned}
(\mathrm{A} 3)= & D \tau^{2} \exp \left[-\frac{\left(t-t^{\prime}\right)}{\tau}\right] c b^{2} \\
& \times\left[a\langle q\rangle_{\mathrm{st}}+b\left\langle q(t) \xi\left(t^{\prime}\right)\right\rangle_{\mathrm{st}}\right] \\
= & D \tau^{2} \exp \left[-\frac{\left(t-t^{\prime}\right)}{\tau}\right] c b^{2} \\
& \times\left[a\langle q\rangle_{\mathrm{st}}+b \int_{0}^{t^{\prime}} d t_{1} \gamma\left(t^{\prime}, t_{1}\right)\left\langle\frac{\delta q(t)}{\delta \xi\left(t_{1}\right)}\right\rangle_{\mathrm{st}}\right] \\
= & D \tau^{2} \exp \left[-\frac{\left(t-t^{\prime}\right)}{\tau}\right] c b^{2}\left[a\langle q\rangle_{\mathrm{st}}-D b^{2}\right. \\
& \left.\times \exp \left[\left(-a+D b^{2}\right)\left(t-t^{\prime}\right)\right]\langle q\rangle_{\mathrm{st}}+O(\tau)\right],(\mathrm{A} 7)
\end{aligned}
$$

where $\left\langle\delta q(t) / \delta \xi\left(t_{1}\right)\right\rangle_{\mathrm{st}}$ is calculated from (A4), recalling that $\xi(t)$ is Gaussian and using a trivial cumulant expansion. The first terms neglected in (A1) are given by the addition of (A6) and (A7):

$$
\begin{aligned}
& D \tau^{2} c b^{2} a(q\rangle_{\mathrm{st}}+D \tau^{2} \exp \left[-\frac{\left(t-t^{\prime}\right)}{\tau}\right] c b^{2} \\
& \quad \times\left\{a-D b^{2}\langle q\rangle\left[1+\exp \left(-a+D b^{2}\right)\left(t-t^{\prime}\right)\right]\right\} .
\end{aligned}
$$

Comparing these terms of order $\tau^{2}$ with the corresponding ones of order $\tau$ in (A1) our criterion of validity for (A1) gives

$$
1>\tau a \text { and } 1>\tau\left(a-2 D b^{2}\right)
$$

since $a>2 D b^{2}$, we finally obtain $\tau<a^{-1}$.

'S. A. Adelman, J. Chem. Phys. 64, 124 (1976).

${ }^{2}$ R. F. Fox, J. Stat. Phys. 16, 259 (1977).

${ }^{3}$ R. F. Fox, J. Math. Phys. 18, 2331 (1977).
${ }^{4}$ P. Hänggi, H. Thomas, H. Grabert, and P. Talkner, J. Stat. Phys. 18, 155 (1978).

${ }^{5}$ M. San Miguel and J. M. Sancho, J. Stat. Phys. 22, 605 (1980).

${ }^{6}$ R. F. Fox, Phys. Rep. C48, 171 (1979).

${ }^{7}$ P. Hänggi and H. Thomas, Phys. Rep. 88, 209 (1982).

${ }^{8}$ N. C. Van Kampen, Phys. Rep. 24, 171 (1976).

${ }^{9}$ For a microscopic approach to non-Markovian processes see: $\mathbf{P}$. Hänggi and H. Thomas, Z. Phys. B 26, 85 (1977); H. Grabert, P. Talkner, P. Hänggi, and Z. Phys. B 26, 398 (1977); H. Grabert, P. Talkner, P. Hänggi, and H. Thomas, Z. Phys. B 29, 273 (1978); S. Grossman, Z. Phys. B 47, 251 (1982).

${ }^{10} \mathrm{~W}$. Horsthemke and R. Lefever, Noise-Induced Transitions, Series in Synergetics (Springer, New York, to be published).

"Stochastic Nonlinear Systems, edited by R. Lefever and L. Arnold (Springer, New York, 1981), papers by W. Horsthemke, R. Lefever, and M. San Miguel and J. M. Sancho.

${ }^{12}$ S. Kabashima, S. Kogura, T. Kawakubo, and T. Okada, J. Appl. Phys. 50, $6296(1979)$

${ }^{13}$ J. M. Sancho, M. San Miguel, H. Yamazaki, and T. Kawakubo, Physica A 116, 5601 (1982).

${ }^{14}$ J. M. Sancho, M. San Miguel, S. L. Katz, and J. D. Gunton, Phys. Rev. A 26, $1589(1982)$.

${ }^{15} \mathrm{~J}$. M. Sancho and M. San Miguel, Z. Phys. B 36, 357 (1980).

${ }^{16}$ E. R. Quabili and M. Karasulu, Ann. Nucl. Energy 6, 133 (1979).

${ }^{17}$ K. Saito, J. Nucl. Sci. Tech. 17, 162 (1980).

${ }^{18}$ S. N. Dixit, P. Zoller, and P. Lambropoulos, Phys. Rev. A 21, 1289 (1980).

${ }^{19}$ P. Zoller, G. Alber, and R. Salvador, Phys. Rev. A 24, 398 (1981).

${ }^{20}$ U. Frisch, in Probabilistic Methods in Applied Mathematics, edited by A. T. Barucha-Reid (Academic, New York, 1968).

${ }^{21}$ R. C. Bourret, Physica 54, 623 (1971).

${ }^{22}$ R. C. Bourret, U. Frisch, and P. Pouquet, Physica 65, 303 (1973).

${ }^{23}$ B. J. West, K. Lindenberg, and V. Seshadri, Physica A 102, 470 (1980);

K. Lindenberg, V. Seshadri, and B. J. West, Phys. Rev. A 22, 2171 (1980). ${ }^{24}$ N. C. Van Kampen, Physica A 102, 489 (1980).

${ }^{25}$ L. Arnold, Stochastic Differential Equations (Wiley, New York, 1974).

${ }^{26}$ It is also possible to think of $\left\langle q(t) q\left(t^{\prime}\right)\right\rangle_{\mathrm{st}}$ as the correlation function of the process defined by $(2.1)-(2.3)$ and arbitrary initial conditions in $t=-\infty$. In this paper we consistently use $t=0$ as the preparation time.

${ }^{27}$ For the MNS process defined by (2.1), (2.3), and (2.7) the two correlation functions which we have defined coincide accidentally. This does not happen for a general nonlinear MNS process.

${ }^{28}$ E. A. Novikov, Zh. Eksp. Teor. Fiz. 47, 1919 (1964) [Sov. Phys. JETP 20, 1290 (1965)].

${ }^{29}$ M. San Miguel and J. M. Sancho, Phys. Lett. A 76, 97 (1980).

${ }^{30}$ Additional contributions to $(2.38)$ and $(2.40)$ in first order in $\tau^{31,32}$ vanish in the examples that we will consider. In these examples $K=0$.

${ }^{31}$ H. Dekker, Phys. Lett. A 90, 26 (1982).

${ }^{32}$ L. Garrido and J. M. Sancho, Physica A 115, 479 (1982).

${ }^{33}$ M. A. Rodriguez and L. Pesquera, J. Nucl. Sci. Tech. 20, 174 (1983).

${ }^{34} \mathrm{The}$ divergence for $a=2 D b^{2}$ is a typical feature of linear multiplicative stochastic processes. It reflects the importance of nonlinear terms in the stability properties. A term proportional to $q^{3}$ in (3.1) would eliminate this divergence.

${ }^{35}$ H. Shibata, Y. Takahasi, and N. Hashitsume, J. Stat. Phys. 17, 171 (1977). ${ }^{36}$ S. Chaturvedi, Phys. Lett. A 90, 444 (1982).

${ }^{37}$ For recent related work see B. J. West and K. Lindenberg, Phys. Lett. A 95, $44(1983)$. 